\title{
A mixed-culture model of a probiotic biofilm control system
}

\author{
Hermann J. Eberl ${ }^{\mathrm{a} *}$, Hassan Khassehkhan ${ }^{\mathrm{a}}$ and Laurent Demaret ${ }^{\mathrm{b}}$ \\ ${ }^{a}$ Department of Mathematics and Statistics, University of Guelph, Guelph, ON, Canada N1G 2W1; \\ ${ }^{b}$ Helmholtz Center Munich, German Research Center for Environment and Health, Institute of \\ Biomathematics and Biometry, 85764 Neuherberg, Germany
}

(Received 24 June 2008; final version received 13 January 2009)

\begin{abstract}
We present a mathematical model and computer simulations for the control of a pathogenic biofilm by a probiotic biofilm. This is a substantial extension of a previous model of control of a pathogenic biofilm by microbial control agents that are suspended in the aqueous bulk phase (H. Khassehkhan and H.J. Eberl, Comp. Math. Meth. Med, 9(1) (2008), pp. 47-67). The mathematical model is a system of double-degenerate diffusion-reaction equations for the microbial biomass fractions probiotics, pathogens and inert bacteria, coupled with convection-diffusion-reaction equations for two growth controlling substrates, protonated lactic acids and hydrogen ions $(\mathrm{pH})$. The latter are produced by the bacteria and become detrimental at high concentrations. In simulation studies, we find that the site of attachment of probiotics in the flow channel is crucial for success and efficacy of the probiotic control mechanism.
\end{abstract}

Keywords: biofilm; probiotics; mathematical model; nonlinear diffusion; simulation

MSC: 92D25; 92C17; 92C50; 65M06

\section{Introduction}

Bacterial biofilms are microbial depositions on surfaces in aqueous environments. The microorganisms attach to the surface and start to produce a gel-like matrix of extracelullar polymers, termed extracellular polymeric substances (EPS) in the biofilm literature (exopolysaccharides). In the EPS layer the bacteria are embedded and protected against harmful environmental impacts, such as washout or antibiotics. In the medical context biofilms are harmful. They are associated with many bacterial infections such as middle ear infections, periodontitis, dental caries, cystic fibrosis pneumonia or infections as a consequence of colonization of surgical implants and surfaces; see Costerton et al. [8] for a more extensive listing.

Diffusive limitation of dissolved substrates is probably the most crucial difference between biofilm communities and the suspended cultures [37], on which microbiologists and mathematical biologists have traditionally focused. It is (at least partially) also the cause of two of the mechanisms that have been identified as reasons for the difficulties in treating biofilm infections with antibiotics [36]: for one, disinfectants penetrate slowly into the biofilm. In many cases, the disinfectants are degraded or weakened upon contact with the bacteria. While the cells in the outer layer of the biofilm are inactivated, the cells in the inner layer remain virtually unharmed over a long time. Thus, the biofilm community

*Corresponding author. Email: heberl@uoguelph.ca 
survives and flourishes. Secondly, since also other substrates become limited in the inner layers of the biofilm, local micro environments can develop in which the living conditions are different than in the surroundings, e.g. with severe oxygen limitation etc. The cells in these regions adapt to the changing living conditions. This can slow down their metabolism and make them less susceptible to disinfectants. Since antibiotic treatment of bacterial biofilms is so much more difficult than treatment of suspended cultures, alternative strategies and means are being developed to control biofilm formation.

An effective mechanical control mechanism is biofilm detachment. It seems to occur when the external shear forces, applied to the biofilm by the flow field in the aqueous environment, exceed the internal tensile strength of the colony [27,28,30]. The latter depends on factors such as biofilm composition and strain specific properties, local growth conditions, internal biochemical reactions, and also the hydrodynamic conditions under which the biofilm was grown $[10,38]$. The former depends on the biofilm morphology, the clustering of the colonies, i.e. their spatial arrangement relative to each other, and of course chiefly on the local hydrodynamic conditions at the biofilm liquid interface $[39,42]$. The internal properties of biofilms are difficult to quantify; therefore mathematical models of detachment often use heuristic and simplifying assumptions or focus solely on the better understood mechanical detachment forces. Mechanical biofilm control is among the most efficient control mechanisms in engineering and industrial applications, but it is probably not appropriate and viable in a medical context.

Another suggested approach for biofilm control is to interfere with the bacterias' cellto-cell communication system by controlling quorum sensing in the biofilm. Mathematical models for such a therapy have been proposed in Anguige et al. [2,3].

A novel alternative to antibiotics are probiotics [7]. Probiotics are defined to be live food ingredients, e.g. bacteria, that confer health benefits to the host when administered in sufficient quantities [19]. Traditionally, probiotics have been used as functional foods, typically in connection with dairy products, but their potential for therapeutic applications is increasingly recognized. The idea is to augment the microbial ecosystem in the body by bacteria that will strengthen the indigenous microflora and worsen the living conditions for the pathogens and keep them from striving. Particular health benefits of probiotics for the host and their clinical applications have been summarized recently in several review articles, e.g. [29,32-34]. Pathogenic bacteria that have been fought with probiotics include Escherichia Coli, Salmonella spp., Shigella spp., and Clostridium difficile. The action of probiotics against pathogens is indirect, e.g. by competing for shared resources or by producing substrates that are detrimental to the pathogens. These effects can be summarized as changing the environmental conditions that the pathogens experience. Important examples are the modification of the $\mathrm{pH}$ value, and the production of antimicrobial substances like bacteriocins, organic acids or hydrogen peroxide [34]. A related concept is to use probiotic strains to control quorum sensing of pathogenic biofilms as investigated experimentally in Valdez et al. [41].

While not all probiotics necessarily form biofilms, [5] argues that colonization of interfaces is in many instances a prerequisit for probiotic efficacy. We propose in this study a mathematical model for one probiotic mechanism of biofilm control, namely the modification of the environmental conditions in the system such that they become less favourable for the pathogen. More specifically we describe a model for the modulation of lactic acid concentration and $\mathrm{pH}$ by a bio-control agent that is more tolerant than the pathogens. In a previous modelling study [22], we described a similar system, consisting of a biofilm forming pathogen and a bio-control agent that was assumed to be suspended in the bulk liquid. Here we propose a mathematical model for a mixed culture biofilm system, 
consisting of a pathogenic biofilm former and a probiotic biofilm former. This requires a substantial extension of the previous model.

\section{Mathematical model}

\subsection{Biofilm model}

The mathematical model that we study here is a major extension of a biofilm control model that was introduced previously in Khassehkhan and Eberl [22], based on the biocontrol model for suspended cultures [6] and the biofilm model [15]. We allow for mixed culture biofilms, formed by pathogen and control agent. Thus, we introduce the biocontrol agent as a second particular biofilm fraction. Moreover, while previously we assumed that dead cells are instantaneously removed from the system, we keep them now as inert biomaterial. This introduces one more particular biomass fraction. The third extension is to explicitly account for local hydrodynamic effects and their contribution to transport of dissolved substrates. Moreover, we aim here at two-dimensional simulations while in the previous study only 1D computations were carried out.

As in Khassehkhan and Eberl [22], we assume that nutrients and other required substances are available to the bacteria in abundance. Biofilm growth and decay are assumed to be controlled only by protonated lactic acids and local $\mathrm{pH}$. Both bacterial species, pathogen and control agent, are in a growth mode if the local concentration of lactic acids is small and if the $\mathrm{pH}$ is sufficiently large. If the lactic acids concentration becomes large or if the $\mathrm{pH}$ becomes small, the bacterial cells are inactivated. Between growth and decay stages lies an extended neutral range with vanishing net growth rate.

The biofilm model that we use is developed in the framework of a density-dependent diffusion-reaction model that has been introduced for a single-species/singlesubstrate biofilm in Eberl et al. [15] and since been studied analytically and numerically in a number of papers on various biofilm processes or for mathematical interest $[9,11-14,16,17,21-23]$.

We adapt the biocontrol model [6] and formulate our model in terms of the dependent variables volume fraction occupied by the pathogen $X$, volume fraction occupied by the biocontrol agent $Y$, volume fraction occupied by inert biomass $Z$, concentration of protonated lactic acids $C$, concentration of hydrogen ions $P$. Note that

$$
\mathrm{pH}=-\log _{10} P
$$

if $P$ is measured in mole. The biofilm model in the two-dimensional computational domain $\Omega$ reads

$$
\left\{\begin{array}{l}
\partial_{t} C=\nabla \cdot\left(D_{C}(M) \nabla C-u C\right)+\alpha_{1} X\left(k_{1}-C\right)+\alpha_{2} Y\left(k_{2}-C\right), \\
\partial_{t} P=\nabla \cdot\left(D_{P}(M) \nabla P-u P\right)+\alpha_{3} C\left(k_{3}-P\right), \\
\partial_{t} X=\nabla \cdot\left(D_{M}(M) \nabla X\right)+\mu_{1} g_{1}(C, P) X \\
\partial_{t} Y=\nabla \cdot\left(D_{M}(M) \nabla Y\right)+\mu_{2} g_{2}(C, P) Y \\
\partial_{t} Z=\nabla \cdot\left(D_{M}(M) \nabla Z\right)-\mu_{1} g_{1}^{-}(C, P) X-\mu_{2} g_{2}^{-}(C, P) Y,
\end{array}\right.
$$

where all constant parameters in (1) are non-negative. The total local volume fraction occupied by biofilm is denoted by

$$
M=X+Y+Z .
$$


The actual biofilm is thus the region $\Omega_{2}(t):=\{x \in \Omega: M(t, x)>0\}$. The region $\Omega_{1}(t):=$ $\{x \in \Omega: M(t, x) \equiv 0\}$ is the surrounding water phase. See also Figure 1. The biofilm liquid interface is thus $\Gamma(t):=\bar{\Omega}_{1}(t) \cap \bar{\Omega}_{2}(t)$.

In (1), the vector $u$ is the hydrodynamic flow velocity of the aqueous phase $\Omega_{1}(t)$. It vanishes in the biofilm phase $\Omega_{2}(t)$. In our simulations it is determined by an analytical approximation to the Navier-Stokes equations that has been developed in Eberl and Sudarsan [14] and is briefly described below in Section 2.2.

The density dependent biomass diffusion coefficient $D_{M}(M)$ in (1) was suggested in Eberl et al. [15] as

$$
D_{M}(M)=\epsilon \frac{M^{a}}{(1-M)^{b}},
$$

where $a, b>1$ and $0<\epsilon \lll 1$. The power law $M^{a}$ guarantees that biomass does not spread if the local density is small; the power law $(1-M)^{-b}$ guarantees that the biomass density remains bounded by the maximum possible cell density, even if inside the biofilm production of new biomass continues. Thus the model includes both finite speed of interface propagation (between biofilm and aqueous phase) and volume filling features. For visualizations of biofilm growth simulations of this density-dependent diffusion model in $2 \mathrm{D}$ or $3 \mathrm{D}$ we refer to $[12,15,18]$.

In the biomass equations, it is implicitly assumed that all particulate substances follow the same spreading mechanism. This is certainly a straightforward assumption for the inert biomass fraction $Z$ which is produced locally by and only if active biomass is present and is moved around with the active biomass when the biofilm expands due to production of new cells. On the other hand, that both active species have the same spreading mechanism strictly speaking needs not be the case. We adopt this assumption here for simplicity, in order to keep the number of parameters small, and for lack of more information; see Eberl and Schraft [13] for a dual species biofilm model with different spreading mechanisms for both bacterial fractions.

In Stewart [37] it is pointed out that the role that diffusion of dissolved substrates plays, in our case $C$ and $P$, constitutes the main difference between biofilm communities and suspended cultures. While in the latter case all cells experience the same conditions, in biofilm communities often dissolved substrates cannot completely penetrate the biofilm or only at a reduced concentration. Moreover, the biofilm matrix poses an increased resistance to diffusing substrates. Therefore, we assume $D_{C}$ and $D_{P}$ to be dependent on the density of the biofilm density, $M$ as well. Due to a lack of more detailed experimental information we make a first order, linearized ansatz,

$$
\begin{aligned}
& D_{C}(M)=D_{C}(0)-M\left(D_{C}(0)-D_{C}(1)\right) \\
& D_{P}(M)=D_{P}(0)-M\left(D_{P}(0)-D_{P}(1)\right)
\end{aligned}
$$

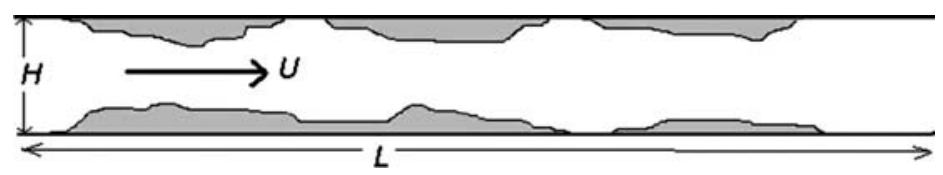

Figure 1. Flow in a long thin channel with aspect ratio $\varepsilon:=H / L \ll 1$ : The biofilm growing on the walls of the channel (grey region $\Omega_{2}(t)$ ) induces a perturbation of the Poiseuille profile that is observed in an empty flow channel. In the white region $\Omega_{1}(t)$ the flow field is described by the flow model (6). 
where $D_{C}(0)$ and $D_{P}(0)$ are the diffusion coefficients in the bulk liquid and $D_{C}(1)$ and $D_{P}(1)$ the diffusion coefficients in a fully developed biofilm. Thus $D_{C}(M)$ and $D_{P}(M)$ are bounded from below and above by known positive constants. Since inside the biofilm $M \approx 1$, diffusion of dissolved substrates in both the aqueous and the biofilm phase behaves essentially like Fickian diffusion.

The reaction terms on the right hand side of (1) stem from Breidt and Flemming [6]. They have the following meaning:

\subsubsection{Protonated lactic acids, $C$}

Lactic acids are produced by both bacterial species until locally a maximum concentration is reached. This is described by first order reaction kinetics. In the model formulation (1) we used two parameters $k_{1}$ and $k_{2}$ to describe this effect, as originally proposed in Breidt and Flemming [6]. However, in Breidt and Flemming [6] it was also suggested based on experiments that $k_{1} \approx k_{2}$, which we will always assume.

\subsubsection{Hydrogen ion concentration, $P$}

The local hydrogen ion concentration increases ( $\mathrm{pH}$ decreases) until a saturation level is reached. This is facilitated by lactic acids and, again, described by first order kinetics.

\subsubsection{Biomass fractions, $X, Y, Z$}

Biomass production is controlled by $C$ and $P$. The growth and inhibition functions $g_{1,2}(C, P)$ in the equations for $X, Y, Z$ are piecewise linear, such that they are positive if both $C$ and $P$ are small, and become negative if one of $C$ and $P$ becomes large. Between the growth and inhibition range there is an extended neutral range. More specifically,

$$
g_{i}(C, P)=\min \left\{1-\frac{C}{H_{1}^{(i)}(C)}, 1-\frac{P}{H_{2}^{(i)}(P)}\right\},
$$

where the auxiliary functions $H_{1}^{(i)}(C)$ and $H_{2}^{(i)}(P)$, for $i=1,2$, are defined by

$$
H_{1}^{(i)}(C)=k_{1}^{(i)} H\left(k_{1}^{(i)}-C\right)+C \cdot H\left(C-k_{1}^{(i)}\right) \cdot H\left(k_{2}^{(i)}-C\right)+k_{2}^{(i)} H\left(C-k_{2}^{(i)}\right)
$$

and

$$
H_{2}^{(i)}(P)=k_{3}^{(i)} H\left(k_{3}^{(i)}-P\right)+P \cdot H\left(P-k_{3}^{(i)}\right) \cdot H\left(k_{4}^{(i)}-P\right)+k_{4}^{(i)} H\left(P-k_{4}^{(i)}\right) .
$$

The function $H$ is defined by

$$
H(x)= \begin{cases}1, & \text { if } x>0 \\ \frac{1}{2}, & \text { if } x=0 \\ 0, & \text { if } x<0\end{cases}
$$

Parameters $k_{1,2,3,4}^{(i)}$ are positive constants with $k_{1}^{(i)}<k_{2}^{(i)}$ and $k_{3}^{(i)}<k_{4}^{(i)}$. The growth function $g_{i}(C, P)$ of (4) is plotted in Figure 2 for a typical set of parameters that were estimated from laboratory experiments in Breidt and Flemming [6]. Moreover, for a probiotic strategy to be successful, the parameters must be such that the probiotics are 


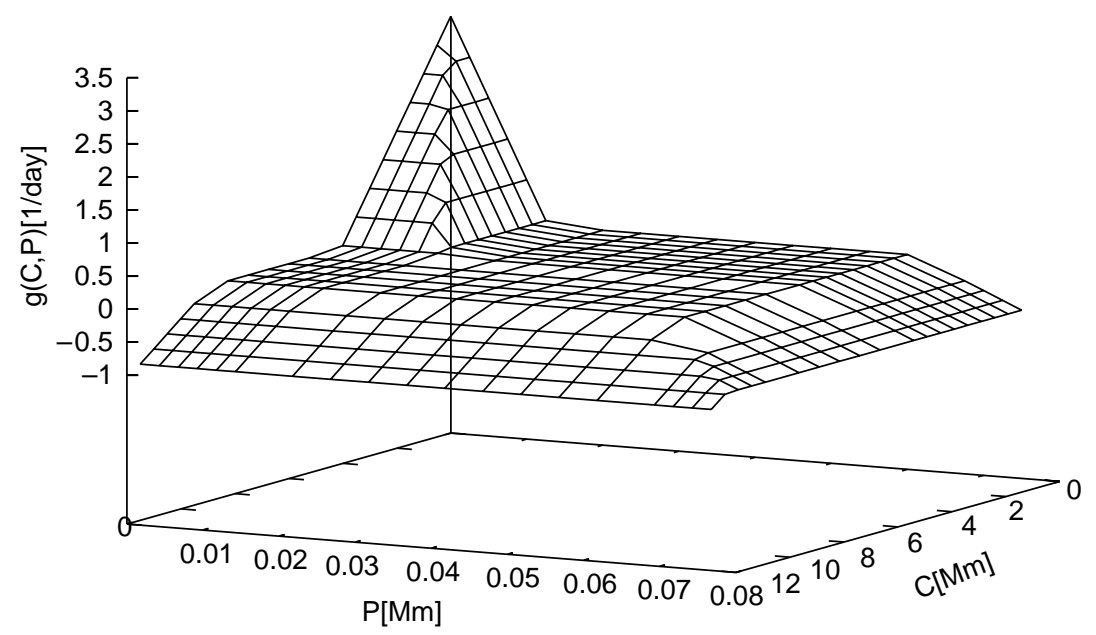

Figure 2. Growth function $\mu g(C, P)$ according to Breidt and Flemming [6]: a piecewise linear function that is positive if both arguments are small and becomes negative if one of the arguments becomes large. Growth and decay phase are separated by a large neutral phase. From Khassehkhan and Eberl [22].

more tolerant to lactic acids and $\mathrm{pH}$ than the pathogen, e.g. $k_{j}^{(1)} \leq k_{j}^{(2)}$ for $j=1, \ldots, 4$ with a strict inequality for at least one $j$.

In (1), we used the notation $g_{i}^{-}(C, P)$ as short hand for the negative part of the growth rate $g_{i}(C, P)$,

$$
g_{i}^{-}(C, P):=\min \left\{0, g_{i}(C, P)\right\}
$$

Note from the equation for $Z$ that, if $g_{i}(C, P)<0$, active biomass $X$ or $Y$, respectively, is converted into inert biomass, one-to-one. We do not distinguish between inactive bacterial species but lump them together into one volume fraction $Z$. If a growth rate is locally negative, the total biomass $M$ is conserved but transformed from active into inert material; if it is positive the total biomass increases.

Model (1) is to be completed by appropriate boundary conditions. We consider a rectangular flow channel of length $L$ and height $H$, i.e. the rectangular computational domain $\Omega=[0, L] \times[0, H]$. On inflow, nonhomogeneous Dirichlet conditions are specified for the dissolved substrates $C$ and $P$, while homogeneous Neumann conditions are specified everywhere else on the boundary of $\Omega$,

$$
\left.P\right|_{x=0}=P_{0},\left.\quad \partial_{n} P\right|_{\partial \Omega \backslash\{x=0\}}=0
$$

and

$$
\left.C\right|_{x=0}=C_{0},\left.\quad \partial_{n} C\right|_{\partial \Omega \backslash\{x=0\}}=0,
$$

where we assume that $P_{0}$ and $C_{0}$ are small enough to be in the growth range for both species

$$
g_{1}\left(C_{0}, P_{0}\right)>0, \quad g_{2}\left(C_{0}, P_{0}\right)>0 .
$$


For the biomass fractions we specify the homogeneous Neumann conditions

$$
\left.\partial_{n} X\right|_{\partial \Omega}=0,\left.\quad \partial_{n} Y\right|_{\partial \Omega}=0,\left.\quad \partial_{n} Z\right|_{\partial \Omega}=0 .
$$

We also specify the following initial conditions for $\mathrm{pH}$ and lactic acids

$$
\left.P\right|_{t=0}=P_{0},\left.\quad C\right|_{t=0}=C_{0} .
$$

Biomass is initially placed in few small discrete pockets along the substratum, i.e. close to $x_{2}=0$ or $x_{2}=H$. We denote this by

$$
\left.X\right|_{t=0}=\left.X_{0}(x) \quad Y\right|_{t=0}=Y_{0}(x) .
$$

Note that the support of $X_{0}$ (and similarly of $Y_{0}$ ) is not connected if the substratum is inoculated with more than one colony initially. For all practical purposes $X_{0}=Y_{0}=0$ for the largest part of $\Omega$. The initial data satisfy furthermore

$$
X_{0}+Y_{0}<\delta<1, \quad 0 \leq X_{0}, \quad 0 \leq Y_{0}
$$

These functions may be continuous or piecewise constant. We furthermore assume normally that initially no inert biomass is in the system

$$
\left.Z\right|_{t=0}=0
$$

Note that with these assumptions, if the initial colonies are separated by species, the biofilm model reduces locally to the single species model that was introduced in Khassehkhan and Eberl [22], as long as the local growth conditions are positive or neutral.

For computational convenience, we non-dimensionalize the model (1). As characteristic time scale we use

$$
T=L^{2} / D_{C}(0)
$$

and as characteristic length scale the length of the rectangular domain in primary flow direction, $L$. The new dimensionless concentration variables are

$$
\hat{C}=\frac{C}{k_{1}}, \quad \hat{P}=\frac{P}{k_{3}} .
$$

We note that the volume fractions $X, Y, Z$, and, thus, $M$, of the biomass constituents were non-dimensional from the start. The new reaction parameters are obtained as

$$
\hat{\mu}_{i}=\frac{\mu_{i}}{T}, \quad \hat{\alpha}_{i}=\frac{\alpha_{i} k_{i}}{T}, \quad \hat{k}_{1,2}^{(j)}=\frac{k_{1,2}^{(j)}}{k_{1}}, \quad \hat{k}_{3,4}^{(j)}=\frac{k_{3,4}^{(j)}}{k_{3}}
$$

and the diffusion coefficients become

$$
\hat{D}_{C}(M)=\frac{D_{C}(M) T}{L^{2}}, \quad \hat{D}_{P}(M)=\frac{D_{P}(M) T}{L^{2}}, \quad \hat{D}_{M}(M)=\hat{\epsilon} \frac{M^{a}}{(1-M)^{b}}, \quad \hat{\epsilon}=\frac{\epsilon T}{L^{2}} .
$$


This leads to the non-dimensional variant of (1)

$$
\left\{\begin{array}{l}
\partial_{t} \hat{C}=\nabla \cdot\left(\hat{D}_{C} \nabla \hat{C}-\hat{u} \hat{C}\right)+\left(\hat{\alpha}_{1} X+\hat{\alpha}_{2} Y\right)(1-\hat{C}), \\
\partial_{t} \hat{P}=\nabla \cdot\left(\hat{D}_{P} \nabla \hat{P}-\hat{u} \hat{P}\right)+\hat{\alpha}_{3} \hat{C}(1-\hat{P}), \\
\partial_{t} X=\nabla \cdot\left(\hat{D}_{M}(M) \nabla X\right)+\hat{\mu}_{1} \hat{g}_{1}(\hat{C}, \hat{P}) X, \\
\partial_{t} Y=\nabla \cdot\left(\hat{D}_{M}(M) \nabla Y\right)+\hat{\mu}_{2} \hat{g}_{2}(\hat{C}, \hat{P}) Y, \\
\partial_{t} Z=\nabla \cdot\left(\hat{D}_{M}(M) \nabla Z\right)-\hat{\mu}_{1} \hat{g}_{1}^{-}(\hat{C}, \hat{P}) X-\hat{\mu}_{2} \hat{g}_{2}^{-}(\hat{C}, \hat{P}) Y .
\end{array}\right.
$$

The non-dimensional model has five parameters fewer than the original version (1): The parameters $k_{1,2,3}$ are not explicitly required anymore, and also the parameters $L$ and $D_{C}(0)$ are fixed to unity. For the remainder of this paper we will use (5), although the $\hat{\text { will be }}$ dropped from the notation.

\subsection{Hydrodynamic model}

The computational domain in our simulations is a rectangular flow channel with a small height:length aspect ratio $\varepsilon:=H / L \ll 1$. The biofilm growing on the channel walls induces a perturbation of the Poiseuille flow field that can be observed for a 'clean' flow channel in the absence of biofilms, cf. Figure 1. A dimensionless parameter that is commonly used to characterize such pipe flow regimes is the Reynolds number

$$
R e=\frac{\rho U H^{2}}{\mu U H}=\frac{\rho U H}{\mu}=\frac{U H}{\nu},
$$

where $\rho$ is the density of the medium and $\mu$ its dynamic viscosity. By $\nu:=\mu / \rho$ we denote the kinematic viscosity. $U$ is a characteristic flow velocity scale. We maintain a constant flow rate $q$ in the flow channel. This is a common flow control mechanism in biofilm experiments that use flow channels or tubular reactors. For the maximum flow velocity of the underlying unperturbed Poiseuille flow we derive $U=3 / 2(q / H)$ and, thus,

$$
\operatorname{Re}=\frac{3}{2} \frac{q}{\nu}
$$

The effect of bulk hydrodynamics in a flow channel on the biofilm is manifested in two clearly distinct phenomena: (i) shear induced deformation and detachment and (ii) convective transport of the dissolved substrates $C$ and $P$ in the flow channel.

The first effect (i) can be expressed by the dimensionless number

$$
\Sigma=\frac{\text { shear }}{\text { material strength }}=\frac{\mu U}{H} \cdot \frac{1}{G}=\frac{\mu^{2}}{\rho \varepsilon} \cdot \frac{R e}{H^{2} G}
$$

where $G$ is the biofilm's apparent elastic modulus, cf. [14]. Measurements for this quantity are sparse in the biofilm literature. In Towler et al. [40] it is found to be around $G \approx 10 \mathrm{~g} \mathrm{~cm}^{-1} \mathrm{~s}^{-2}$; in Klapper et al. [24] it ranges between 10 and $1000 \mathrm{~g} \mathrm{~cm}^{-1} \mathrm{~s}^{-2}$. We use these values as order of magnitude estimates. For water typical values of $\mu$ and $\rho$ are $\mu \approx 10^{-2} \mathrm{~g} \mathrm{~cm}^{-1} \mathrm{~s}^{-1}, \rho=1 \mathrm{~g} \mathrm{~cm}^{-3}$. In our simulations later on we will consider slow flows with $R e=2 \times 10^{-3}$ and domains with $10^{-2} \mathrm{~cm}<H<1 \mathrm{~cm}, \varepsilon \approx 0.1$. 
Then, $\Sigma<<1$ and deformations of the biofilm matrix indeed can be neglected, as it is the case in our bioflm model.

The second effect (ii) is expressed in terms of the dimensionless Peclet number

$$
P e=\frac{\text { convective transport }}{\text { diffusive transport }}=\frac{U L}{D}=\frac{U H}{\nu} \cdot \frac{L}{H} \cdot \frac{\nu}{D}=R e \cdot \frac{1}{\varepsilon} \cdot S c
$$

where $D$ is the diffusion coefficient of the transported substrate in the flowing liquid and $S c=\nu / D$ is the Schmitt number that approximates the ratio of the (fluid) momentum diffusivity and (substrate) mass diffusivity. This is a constant that depends on both the substrate and the fluid. For small molecules like oxygen in water one has large values for Sc, e.g. $\nu \approx 0.01 \mathrm{~cm}^{2} \mathrm{~s}^{-1}$ and $D \approx 2 \times 10^{-5} \mathrm{~cm}^{2} \mathrm{~s}^{-1}$, hence, $S c \approx 10^{3}$. Since also $\varepsilon<1$ is small, we obtain $1 / \varepsilon \gg 1$ and, thus, $P e \gg R e$. Hence convective contribution to substrate transport can be substantial, even if $R e<<1$. This was also shown in numerical simulations in Eberl and Sudarsan [14]. In the case of our biocontrol model (1) with the boundary conditions specified above, the effect of the flow field is to remove detrimental substrates $C$ and $P$ from the channel and to replenish the system by supplying fresh medium in growth conditions, i.e. with low concentrations $C_{0}$ and $P_{0}$.

A mathematical model for the calculation of the flow field $u$ in (1) that is consistent with the assumptions discussed above has been introduced in Eberl and Sudarsan [14] for biofilms growing in long skinny channels. This is the thin liquid film flow model, which is frequently used in lubrication theory [4,35]. It reads

$$
\left\{\begin{array}{l}
0=-\frac{\partial p}{\partial x_{1}}+\mu \frac{\partial u_{1}}{\partial x_{2}^{2}} \\
0=-\frac{\partial p}{\partial x_{2}} \\
0=\frac{\partial u_{1}}{\partial x_{1}}+\frac{\partial u_{2}}{\partial x_{2}}
\end{array}\right.
$$

in the liquid region $\Omega_{1}(t)$, while $u \equiv 0$ in the biofilm region $\Omega_{2}(t)$. In (6) $p$ is the pressure field and $\left(u_{1}, u_{2}\right)^{T}=u$ is the flow velocity vector. At the biofilm/water interface in the flow channel and at the walls of the channel where they are not covered by biofilm, the no-slip condition $u=0$ is enforced. The flow is driven by prescribing the flow rate $q$, or, which is equivalent, the Reynolds number $R e$.

The system of partial differential equations (6) can be solved analytically in $\Omega_{1}(t)$ with elementary techniques. This allows for very fast calculations in the numerical simulation. The solution is not repeated here. Instead, we refer the reader to Eberl and Sudarsan [14]. We note that the solution of (6) is an $\mathcal{O}\left(\varepsilon^{2}\right)$ approximation of the Stokes equations that are commonly used (and numerically solved) to describe low Reynolds number flow, $R e<1$.

\subsection{Numerical discretization scheme}

The numerical discretization of (1) follows the strategy that was introduced in Eberl and Demaret [12] for a single-species biofilm model. The critical component of every discretization scheme is the treatment of the biomass equations while standard methods can be used to compute $C$ and $P$. The key feature in our numerical treatment is a non-local (in time) discretization of the nonlinearities of the model, according to the definition of Nonstandard Finite Difference Schemes [1,26]. In the time-step $t_{n} \rightarrow t_{n+1}$ we use for the 
density-dependent diffusive flux

$$
D_{M}(M) \cdot \nabla X \approx D_{M}\left(M\left(t_{n}, \cdot\right)\right) \cdot \nabla X\left(t_{n+1}, \cdot\right)
$$

(and similarly for $Y, Z, C, P$ ), for the convective flux

$$
u C \approx u\left(t_{n}, \cdot\right) C\left(t_{n+1}, \cdot\right)
$$

(similar for $P$ ). The nonlinear reaction terms can be straightforwardly treated in the same manner.

For the numerical realization we introduce a uniform rectangular grid of $n_{1} \times n_{2}$ cells with mesh size $\Delta x$. The substrate concentrations $C$ and $P$ and the biomass fractions $X, Y, Z$ are approximated in the cell centres. We denote by $C_{i, j}^{k}$ the numerical approximation of $C\left(t_{k},(i-1 / 2) \Delta x,(j-1 / 2) \Delta x\right)$ etc. Moreover, we denote the (variable) time step size $\tau^{k}:=t_{k+1}-t_{k}$.

In order to be able to write the discretized equations in standard matrix-vector notation, we introduce a bijective grid ordering

$$
\pi:\left\{1, \ldots, n_{1}\right\} \times\left\{1, \ldots, n_{2}\right\} \rightarrow\left\{1, \ldots, n_{1} n_{2}\right\}, \quad(i, j) \mapsto \pi(i, j)
$$

We will use the simple lexicographical ordering $\pi=(i-1) n_{1}+j$. The vectors $\mathcal{X}, \mathcal{Y}, \mathcal{Z}, \mathcal{C}, \mathcal{P}$ are then defined by their coefficients as $\mathcal{X}_{p}=X_{i, j}$ with $p=\pi(i, j)$, etc. For the discretization of the diffusion operator we introduce for every grid point the grid neighbourhood $\mathcal{N}_{p}$ as the index set of direct neighbour points. For grid points $(i, j)$ in the interior of the domain $1<i<n_{1}, 1<j<n_{2}$ this is the set with four elements $N_{p(i, j)}=\{p(i \pm 1, j), p(i, j \pm 1)\}$. In the case of grid cells that are aligned with boundaries of the domain, i.e. $i=1$ or $i=n_{1}$ or $j=1$ or $j=n_{2}$, this definition of the grid neighbourhood leads to ghost cells outside the domain. These can be eliminated in the usual way by substituting the boundary conditions.

The resulting discretization scheme reads

$$
\begin{aligned}
\mathcal{C}_{p}^{k+1}=\mathcal{C}_{p}^{k} & +\frac{\tau^{k}}{2 \Delta x^{2}} \sum_{s \in \mathcal{N}_{p}}\left(D_{C}\left(\mathcal{M}_{s}^{k}\right)+D_{C}\left(\mathcal{M}_{p}^{k}\right)\right)\left(\mathcal{C}_{s}^{k+1}-\mathcal{C}_{p}^{k+1}\right)+ \\
& - \text { convection } \\
& +\tau^{k}\left(1-\mathcal{C}_{p}^{k+1}\right)\left[\alpha_{1} \mathcal{X}_{p}^{k}+\alpha_{2} \mathcal{X}_{p}^{k}\right] \\
\mathcal{P}_{p}^{k+1}=\mathcal{P}_{p}^{k} & +\frac{\tau^{k}}{2 \Delta x^{2}} \sum_{s \in \mathcal{N}_{p}}\left(D_{P}\left(\mathcal{M}_{s}^{k}\right)+D_{P}\left(\mathcal{M}_{p}^{k}\right)\right)\left(\mathcal{P}_{s}^{k+1}-\mathcal{P}_{p}^{k+1}\right)+ \\
& -\operatorname{convection}^{k} \\
& +\tau^{k} \alpha_{3} \mathcal{C}_{p}^{k}\left(1-\mathcal{P}_{p}^{k+1}\right)
\end{aligned}
$$




$$
\begin{aligned}
\mathcal{X}_{p}^{k+1}=\mathcal{X}_{p}^{k} & +\frac{\tau^{k}}{2 \Delta x^{2}} \sum_{s \in \mathcal{N}_{p}}\left(D_{M}\left(\mathcal{M}_{s}^{k}\right)+D_{M}\left(\mathcal{M}_{p}^{k}\right)\right)\left(\mathcal{X}_{s}^{k+1}-\mathcal{X}_{p}^{k+1}\right) \\
& +\tau^{k} \mu_{1} g_{1}\left(\mathcal{C}_{p}^{k}, \mathcal{P}_{p}^{k}\right) \mathcal{X}_{p}^{k+1} \\
\mathcal{Y}_{p}^{k+1}=\mathcal{Y}_{p}^{k} & +\frac{\tau^{k}}{2 \Delta x^{2}} \sum_{s \in \mathcal{N}_{p}}\left(D_{M}\left(\mathcal{M}_{s}^{k}\right)+D_{M}\left(\mathcal{M}_{p}^{k}\right)\right)\left(\mathcal{Y}_{s}^{k+1}-\mathcal{Y}_{p}^{k+1}\right) \\
& +\tau^{k} \mu_{2} g_{2}\left(\mathcal{C}_{p}^{k}, \mathcal{P}_{p}^{k}\right) \mathcal{Y}_{p}^{k+1} \\
\mathcal{Z}_{p}^{k+1}=\mathcal{Z}_{p}^{k}+ & \frac{\tau^{k}}{2 \Delta x^{2}} \sum_{s \in \mathcal{N}_{p}}\left(D_{M}\left(\mathcal{M}_{s}^{k}\right)+D_{M}\left(\mathcal{M}_{p}^{k}\right)\right)\left(\mathcal{Z}_{s}^{k+1}-\mathcal{Z}_{p}^{k+1}\right) \\
& -\tau^{k}\left(\mu_{1} g_{1}^{-}\left(\mathcal{C}_{p}^{k}, \mathcal{P}_{p}^{k}\right) \mathcal{X}_{p}^{k+1}+\mu_{2} g_{2}^{-}\left(\mathcal{C}_{p}^{k}, \mathcal{P}_{p}^{k}\right) \mathcal{Y}_{p}^{k+1}\right)
\end{aligned}
$$

Note that in (9) and (10) the discretization of the convective flux is not explicitly specified. For all practical purposes any standard scheme from the Finite Volume Method literature can be used, e.g. those in Morton [25]. In complying with the rule of constructing nonstandard-finite difference schemes to keep the order of the discrete difference approximation the same as the order of the continuous derivative [26], we use a simple 1st order upwinding method.

For non-homogeneous Dirichlet or Neumann boundary cells, additional boundary contributions are obtained on the right-hand side in the usual manner.

The new flow variables $u^{k+1}$ are computed after the new values for $\mathcal{X}, \mathcal{Y}, \mathcal{Z}$ are available, i.e. for the new biofilm structure. We use the analytical solution to (6) to approximate the flow velocities on the same uniform grid that was used for the other variables, albeit in a staggered fashion. The pressure is evaluated in the cell-centres like the dissolved and the particulate substrates. The flow velocity components are evaluated on the cell edges, i.e. $u_{1 ; i, j}$ approximates the primary flow velocity in the point ( $i \Delta x,(j-1 / 2) \Delta x)$, and $u_{2 ; i, j}$ approximates the secondary flow velocity in $((i-1 / 2) \Delta x, j)$.

After rearranging terms, Equations (9)-(13) can be written as five sparse, structurally symmetric but not symmetric linear system that need to be solved in every time-step. The numerical discretization method is rather standard for the non-degenerate equations describing the dissolved substrates $C$ and $P$. More interesting is the computation of the biomass fractions $\mathcal{X}, \mathcal{Y}, \mathcal{Z}$ because of the degenerate and fast diffusion effects.

In Eberl and Demaret [12] it was shown that this discretization method applied to a single species biofilm model renders the following properties of the solution of the underlying continuous model: (i) positivity, (ii) boundedness by 1, (iii) finite speed of interface propagation, (iv) a discrete interface condition that corresponds to the continuous interface condition of the partial differential equations (PDE), (v) a sharp interface in the numerical solution with only weak interface smearing effects, (vi) monotonicity of solutions near the interface and well-posedness of the merging of colonies (no spurious oscillations).

Proposition 1. These properties carry over to the multi-species biofilm system investigated here. 
Proof. To see this we rewrite (11)-(13) in matrix-vector form

$$
\left\{\begin{array}{l}
\left(\mathcal{I}-\tau^{k} \mathcal{D}^{k}-\tau^{k} \mathcal{G}_{X}^{k}\right) \mathcal{X}^{k+1}=\mathcal{X}^{k} \\
\left(\mathcal{I}-\tau^{k} \mathcal{D}^{k}-\tau^{k} \mathcal{G}_{Y}^{k}\right) \mathcal{Y}^{k+1}=\mathcal{Y}^{k} \\
\left(\mathcal{I}-\tau^{k} \mathcal{D}^{k}\right) \mathcal{Z}^{k+1}=\mathcal{Z}^{k}-\tau^{k} \mathcal{G}_{X}^{-, k} \mathcal{X}^{k+1}-\tau^{k} \mathcal{G}_{Y}^{-, k} \mathcal{Y}^{k+1}
\end{array}\right.
$$

where $\mathcal{I}$ is the $n_{1} n_{2} \times n_{1} n_{2}$ identity matrix, the matrix $\mathcal{D}^{k}$ contains the diffusive contribution, i.e. $\quad\left(\mathcal{D}^{k}\right)_{p, p}=-1 /\left(2 \Delta x^{2}\right) \sum_{s \in \mathcal{N}_{p}}\left(D_{M}\left(\mathcal{M}_{s}^{k}\right)+D_{M}\left(\mathcal{M}_{p}^{k}\right)\right) \quad$ and $\quad\left(\mathcal{D}^{k}\right)_{p, s}=\left(1 /\left(2 \Delta x^{2}\right)\right) \times$ $\left(D_{M}\left(\mathcal{M}_{s}^{k}\right)+D_{M}\left(\mathcal{M}_{p}^{k}\right)\right)$ for component $p$ and $s \in \mathcal{N}_{p}$, and the diagonal matrices $\mathcal{G}_{X, Y}$ and $\mathcal{G}_{X, Y}^{-, k}$ contain the reaction terms $\mathcal{G}_{X}^{k}=\operatorname{diag}_{p=1, \ldots, n_{1} n_{2}}\left(\mu_{1} g_{1}\left(\mathcal{C}_{p}^{k}, \mathcal{P}_{p}^{k}\right)\right), \mathcal{G}_{Y}^{k}=\operatorname{diag}_{p=1, \ldots, n_{1} n_{2}}$ $\left(\mu_{2} g_{2}\left(\mathcal{C}_{p}^{k}, \mathcal{P}_{p}^{k}\right)\right), \mathcal{G}_{X}^{-, k}=\operatorname{diag}_{p=1, \ldots, n_{1} n_{2}}\left(\mu_{1} g_{1}^{-}\left(\mathcal{C}_{p}^{k}, \mathcal{P}_{p}^{k}\right)\right), \mathcal{G}_{Y}^{k}=\operatorname{diag}_{p=1, \ldots, n_{1} n_{2}}\left(\mu_{2} g_{2}^{-}\left(\mathcal{C}_{p}^{k}, \mathcal{P}_{p}^{k}\right)\right)$.

The positivity of each biomass fraction $\mathcal{X}, \mathcal{Y}, \mathcal{Z}$ individually follows with exactly the same argument that was used in Eberl and Demaret [12] for the single species model: the matrix $\mathcal{D}^{k}$ is (at least) weakly diagonally dominant with negative diagonal entries and positive off-diagonal entries. If $\tau^{k}$ is chosen such that the diagonal matrices $\mathcal{I}-\tau^{k} \mathcal{G}_{X}^{k}$ and $\mathcal{I}-\tau^{k} \mathcal{G}_{Y}^{k}$ are positive, then the system matrices in (14) are diagonally dominant with positive diagonal entries and negative off-diagonal entries. Thus, they are $M$-matrices [20] and, therefore, their inverses are non-negative. Hence, if $\mathcal{X}^{k}, \mathcal{Y}^{k}, \mathcal{Z}^{k}$ are non-negative, then so are $\mathcal{X}^{k+1}=\left(\mathcal{I}-\tau^{k} \mathcal{D}^{k}-\tau^{k} \mathcal{G}_{X}^{k}\right)^{-1} \mathcal{X}^{k}, \mathcal{Y}^{k+1}=\left(\mathcal{I}-\tau^{k} \mathcal{D}^{k}-\tau^{k} \mathcal{G}_{Y}^{k}\right)^{-1} \mathcal{Y}^{k}$ and finally $\mathcal{Z}^{k+1}=\left(\mathcal{I}-\tau^{k} \mathcal{D}^{k}\right)^{-1}\left(\mathcal{Z}^{k}-\tau^{k} \mathcal{G}_{X}^{-, k} \mathcal{X}^{k+1}-\tau^{k} \mathcal{G}_{Y}^{-, k} \mathcal{Y}^{k+1}\right)$. The sufficient time-step restriction for $\tau^{k}$ depends on the growth rates only. In fact $\tau^{k}$ is bounded by the reciprocal of the maximum growth rate, $\max _{i=1,2}\left\{1 / \mu_{i} g_{i}(0,0)\right\}$. This is the time-scale for growth. Since we want to describe the time-evolution of the biofilm, i.e. resolve a time-scale not bigger than the time-scale for growth, the restriction placed here on the time-step is not critical for practical purposes.

Having positivity of the individual components of the numerical solution $\mathcal{X}, \mathcal{Y}, \mathcal{Z}$ established, we can deduce the remaining properties with the following trick: adding Equations (11)-(13) [or the equations in (14) for that matter], we obtain for $\mathcal{M}:=\mathcal{X}+\mathcal{Y}+\mathcal{Z}$ the (component wise) inequality

$$
\begin{aligned}
\mathcal{M}^{k} & =\left(\mathcal{I}-\tau^{k} \mathcal{D}^{k}\right) \mathcal{M}^{k+1}-\tau^{k}\left(\mathcal{G}_{X}^{k}-\mathcal{G}_{X}^{-, k}\right) \mathcal{X}^{k+1}-\tau^{k}\left(\mathcal{G}_{Y}^{k}-\mathcal{G}_{Y}^{-, k}\right) \mathcal{Y}^{k+1} \\
& \geq\left(\mathcal{I}-\tau^{k} \mathcal{D}^{k}-\tau^{k} \mathcal{G}^{+, k}\right) \mathcal{M}^{k+1}
\end{aligned}
$$

where the diagonal matrices $\mathcal{G}_{X}^{k}-\mathcal{G}_{X}^{-, k}$ and $\mathcal{G}_{Y}^{k}-\mathcal{G}_{Y}^{-, k}$ are non-negative and $\mathcal{G}^{+, k}:=$ $\max \left\{\mathcal{G}_{X}^{k}-\mathcal{G}_{X}^{-, k}, \mathcal{G}_{Y}^{k}-\mathcal{G}_{Y}^{-, k}\right\} \geq 0$ entry-wise. We denote by $\tilde{\mathcal{M}}^{k+1}$ the solution of the associated linear system

$$
\left(\mathcal{I}-\tau^{k} \mathcal{D}^{k}-\tau^{k} \mathcal{G}^{+, k}\right) \tilde{\mathcal{M}}^{k+1}=\mathcal{M}^{k}
$$

The system matrix $\mathcal{I}-\tau^{k} \mathcal{D}^{k}-\tau^{k} \mathcal{G}^{+, k}$ is again an $M$-matrix, i.e. its inverse has only non-negative entries. Then it follows from (15) and (16) immediately that $\tilde{\mathcal{M}}^{k+1} \geq \mathcal{M}^{k+1}$.

Moreover, as a solution of (16), $\tilde{\mathcal{M}}$ is also a numerical solution of a single species biofilm equation, as studied in Eberl and Demaret [12]. Thus, it has the properties (i) -(vi) above. By comparison, it follows, again due to positivity, immediately that also $\mathcal{X}^{k+1}$, $\mathcal{Y}^{k+1}, \mathcal{Z}^{k+1}$ are bounded by 1 , have a finite speed of interface propagation, show only weak interface smearing effects and are oscillation free. 
We use the stabilized bi-conjugated gradient method to solve the linear system of (9)-(13) in every time-step of the simulation. This is the computationally most expensive part of the numerical algorithm and has been prepared for parallel execution on shared memory architectures using OpenMP. The code has been implemented in platform portable Fortran 90. To solve the linear system in every time-step we use selected routines of the reverse communication Fortran 77 source code library SPARSKIT [31]. The code was compiled and tested onthe following computer system: an off-the-shelf Intel Pentium 4 based Notebook under Windows XP using the gnu95 compiler as well as the Salford FTN95 compiler; a desktop workstation with two Intel Xeon processors under Red Hat Linux using the Fortran compiler; a SGI Altix 330 compute server with 16 Intel Itanium 2 processors under SUSE Linux using the Intel Fortran compiler. The simulations that are reported in this paper have been conducted on the latter machine, in most cases using 2 or 4 processors per compute job, after testing for parallel speed-up efficiency.

\section{Simulation experiment}

For our numerical simulation we consider a two-dimensional rectangular domain of size $\Omega=L \times H$, which we discretize by a uniform grid of $600 \times 60$ computational cells. A pressure driven creeping flow $\left(\operatorname{Re}=2 \times 10^{-3}\right)$ is applied along the length side of the domain. The model parameters that were used in this study are given in Table 1.

Table 1. Dimensionless model parameters used in the numerical simulations.

\begin{tabular}{llc}
\hline Parameter & Symbol & Value \\
\hline Length of domain & $L$ & 1 \\
Height of domain & $H$ & 0.1 \\
Aspect ratio & $\varepsilon$ & $H / L$ \\
Reynolds number & $R e$ & $2 \times 10^{-3}$ \\
Maximum growth rate, pathogens & $\mu_{1}$ & 267 \\
Maximum growth rate, probiotics & $\mu_{2}$ & 267 \\
Acid production rate of pathogens & $\alpha_{1}$ & $4.44 \times 10^{5}$ \\
Acid production rate of probiotics & $\alpha_{2}$ & $4.44 \times 10^{5}$ \\
Hydrogen ion production rate & $\alpha_{3}$ & $8.89 \times 10^{5}$ \\
Pathogen growth kinetics & $k_{1}^{(1)}$ & 0.30 \\
& $k_{2}^{(1)}$ & 0.40 \\
& $k_{3}^{(1)}$ & 0.30 \\
& $k_{4}^{(1)}$ & 0.40 \\
Probiotic growth kinetics & $k_{1}^{(2)}$ & 0.35 \\
& $k_{2}^{(2)}$ & 0.45 \\
& $k_{3}^{(2)}$ & 0.35 \\
Bulk concentration, lactic acids (normalized) & $k_{4}^{(2)}$ & 0.45 \\
Bulk concentration, hydrogen ions (normalized) & $C_{0}$ & 0.1 \\
Diffusion coefficient of $C$ in biofilm & $P_{0}$ & 0.1 \\
Diffusion coefficient of $C$ in water & $D_{C}(1)$ & 0.9 \\
Diffusion coefficient of $P$ in biofilm & $D_{C}(0)$ & 1 \\
Diffusion coefficient of $P$ in water & $D_{P}(1)$ & 0.72 \\
Biofilm interface exponent & $D_{P}(0)$ & 0.8 \\
Biofilm threshold exponent & $a$ & 4 \\
Biofilm motility coefficient & $b$ & 4 \\
\hline & $\epsilon$ & $6.25 \times 10^{-10}$ \\
\hline
\end{tabular}



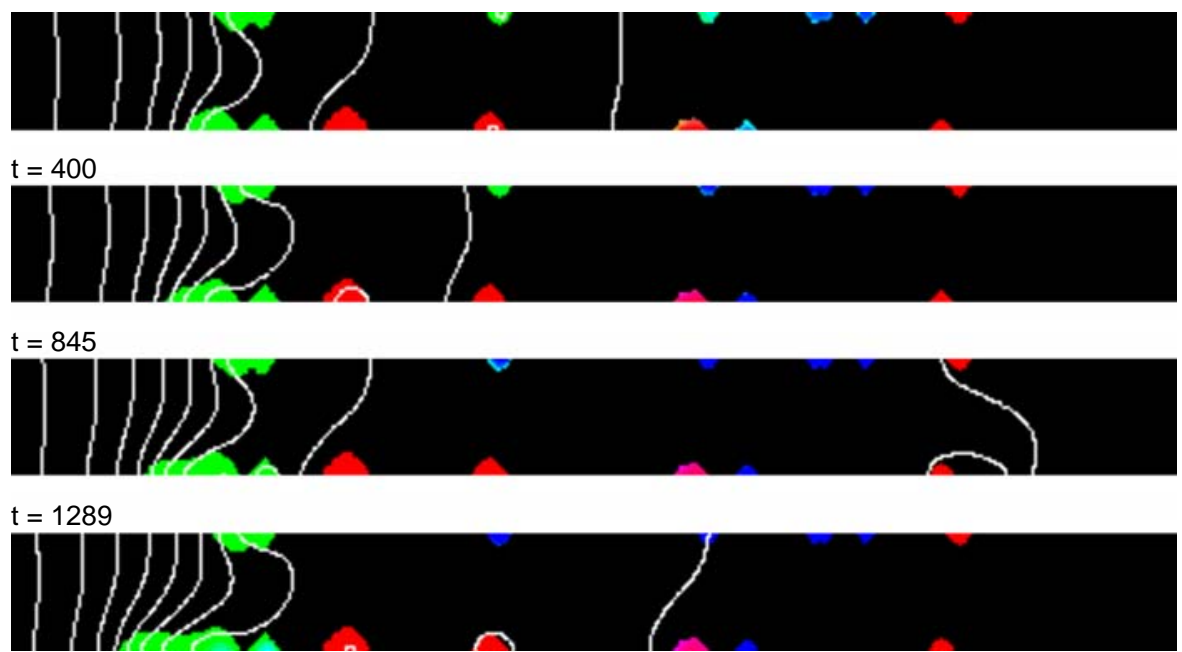

$t=1733$

Figure 3. Population dynamics in a mixed-culture biofilm system of probiotics (red) and pathogens (green); inert biomass is depicted in blue. The flow direction is from left to right. Shown are the volume fractions for the indicated time levels encoded as RGB channels, i.e. colour intensities reflect biomass densities. Also shown are equidistant iso-concentration lines for lactic acids $C$.

Initially biomass is placed in discrete inoculation sites on the substratum, i.e. in few grid cells along the length sides of the domain, $y=0(j=1)$ or $y=H\left(j=n_{2}\right)$. The number of inoculation sites is specified for both active biomass fractions, probiotic ( 5 sites) and pathogen (10 sites). Their locations are chosen randomly. Also the initial biomass density in these inoculation sites is chosen randomly, in a given interval. Initially no inert biomass is assumed in the system, $Z_{0} \equiv 0$.

A typical model simulation is illustrated in Figure 3. Initially, the entire domain lies under growth conditions. In a first transient phase the active biomass densities increase locally without much spatial spreading. Eventually, as locally the maximum cell density is approached, $X \approx 1$ or $Y \approx 1$, the biofilm colonies start to expand. All active biomass in the system produces lactic acids, which in turn increases the hydrogen ion concentration. The flow field carries both detrimental substrates downstream where they attain higher concentrations than in the inflow region. Thus, the active biomass colonies closer to the entrance region live under more favourable conditions than the ones in the outflow region of the domain. Accordingly they develop faster and bigger. Initially separated neighbouring colonies eventually can merge into a single, larger colony. In the centre of the domain and in the downstream region biofilm growth is inhibited rather quickly. Probiotics remain largely in a neutral no-growth mode. Inert biomass is first formed downstream by the pathogens which are less tolerant against lactic acids and $\mathrm{pH}$. Eventually also in the pathogenic biofilm colonies in the upstream regions conversion of active to inert biomass takes place. The last plotted time-step shows how in one colony both processes, killing and production of new biomass can happen simultaneously. More specific, the colony grows upstream toward the region of better conditions (lower $C, P$ ), while in the downstream end of these colonies, active biomass is converted into dead biomass. 


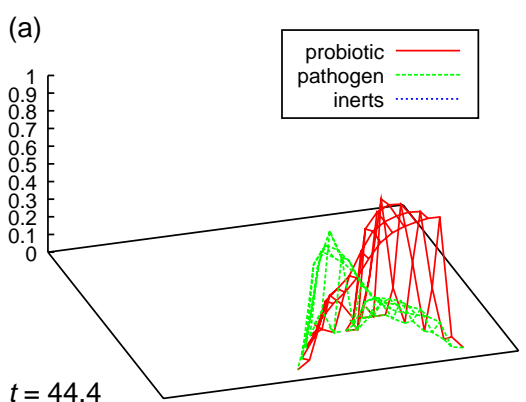

(c)

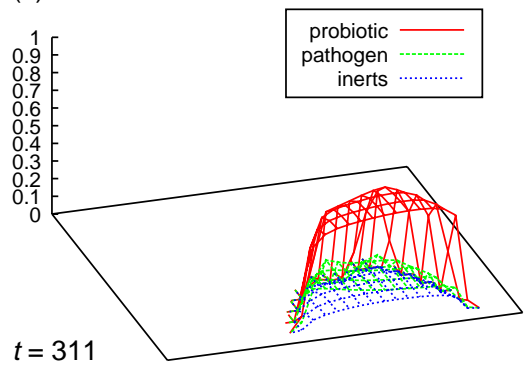

(e)

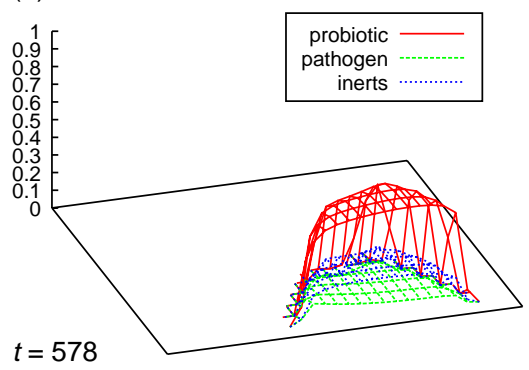

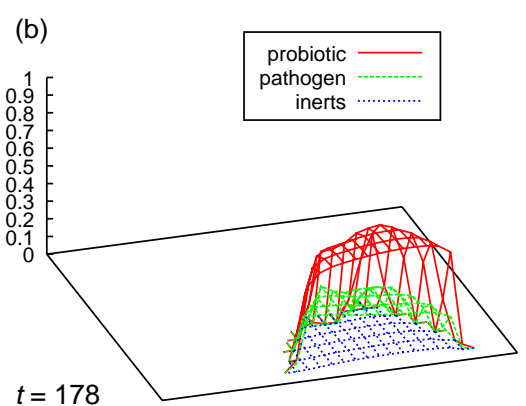

(d)

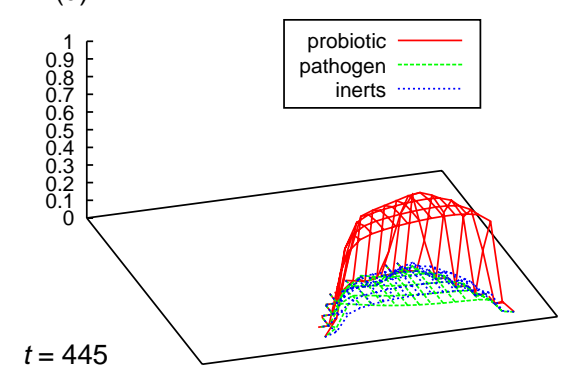

(f)

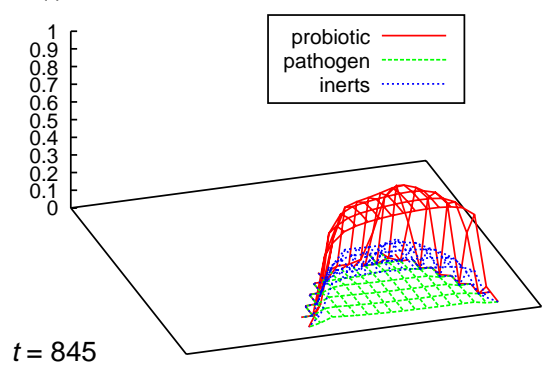

Figure 4. Biomass density surfaces of pathogens $X(t, \cdot)$ [green], probiotics $Y(t, \cdot)$ [red], and inerts $Z(t, \cdot)$ [blue] in a mixed culture colony for selected time-instances.

At the lower substratum, approximately in the middle of the domain, a small colony can be found that is formed by probiotics and pathogens, as a consequence of the random choice of inoculation sites and subsequent merger of two different colonies. The evolution of this mixed-culture colony is visualized in more detail in the Figure 4 where the volume fractions $X, Y$ and $Z$ are shown as surface plots for selected time instances. As time progresses it can be observed how the pathogen is continuously transformed into inert biomass while the probiotics survive. All other colonies remain single-species throughout, as a consequence of the sparse random inoculation.

It should be noted that in the simulation depicted here, the eradication of the pathogen is due to an increased concentration of lactic acids. The $\mathrm{pH}$ remains in the growth range upstream and in the neutral no growth/no decay range further downstream.

We repeated the simulation described above several times, with different randomly chosen inoculation sites (i.e. inital data). In Figure 5 we plot the total biomass of probiotic, 

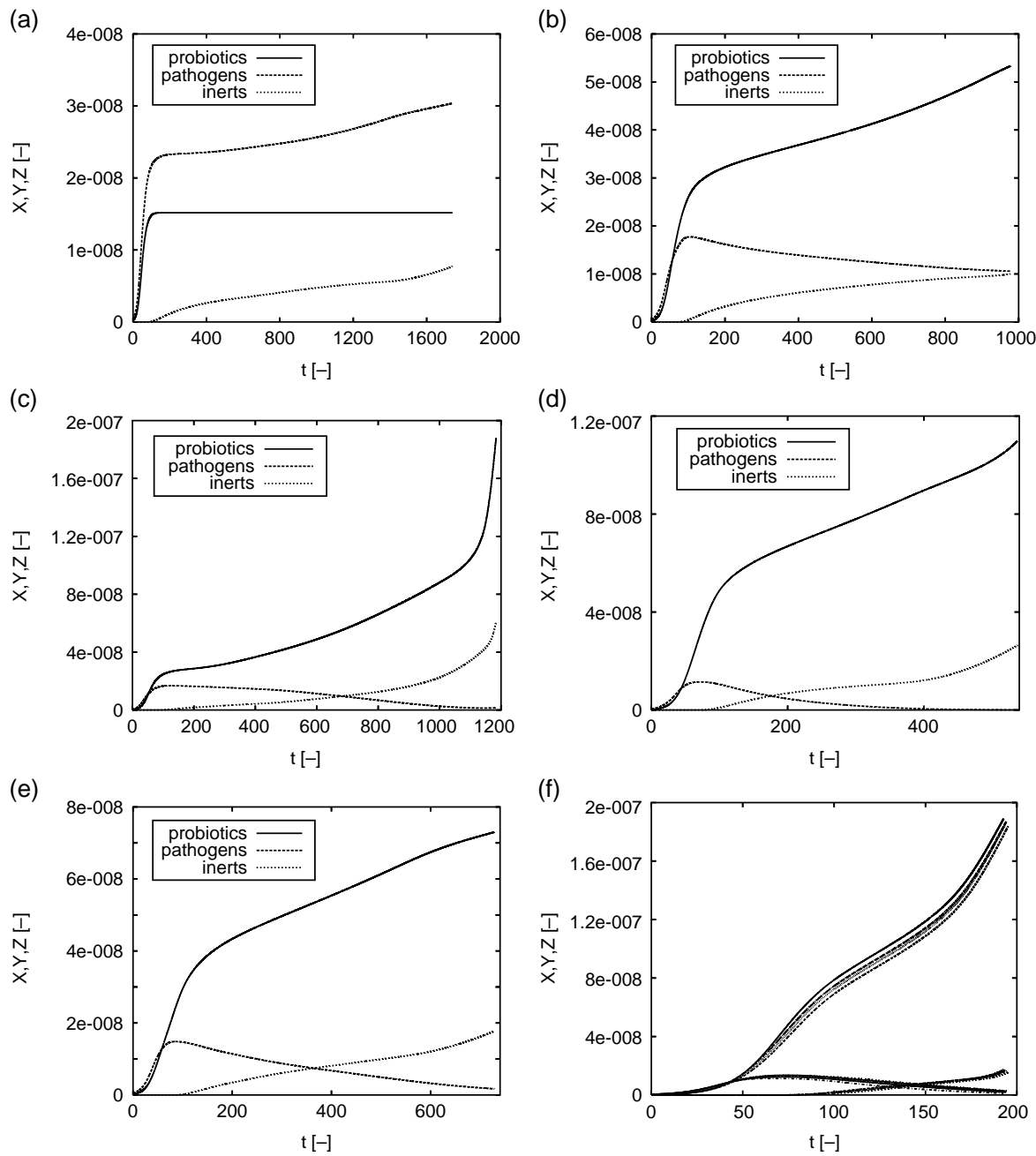

Figure 5. (a)-(e) Biomass of pathogens $X$, probiotics $Y$, and inerts $Z$ for randomly chosen inoculation sites. (f) five simulations with some probiotics placed in the upstream region: $Y$ is increasing, $X$ eventually declines, $Z$ increases as $X$ decreases.

pathogen and inert biomass in the system, i.e. the functions

$$
X_{\text {total }}(t)=\int_{\Omega} X(t, x, y) \mathrm{d} x \mathrm{~d} y, \quad Y_{\text {total }}(t)=\int_{\Omega} Y(t, x, y) \mathrm{d} x \mathrm{~d} y, \quad Z_{\text {total }}(t)=\int_{\Omega} Z(t, x, y) \mathrm{d} x \mathrm{~d} y
$$

for five such runs, namely (a)-(e). The size of the biofilm

$$
\omega(t)=\frac{\int_{\Omega_{2}(t)} \mathrm{d} x \mathrm{~d} y}{\int_{\Omega} \mathrm{d} x \mathrm{~d} y}
$$

is plotted in Figure 6(a).

For the given parameter sets and inflow concentrations the model shows different behaviour across these five cases. In case (a) the probiotic population reaches quickly a 

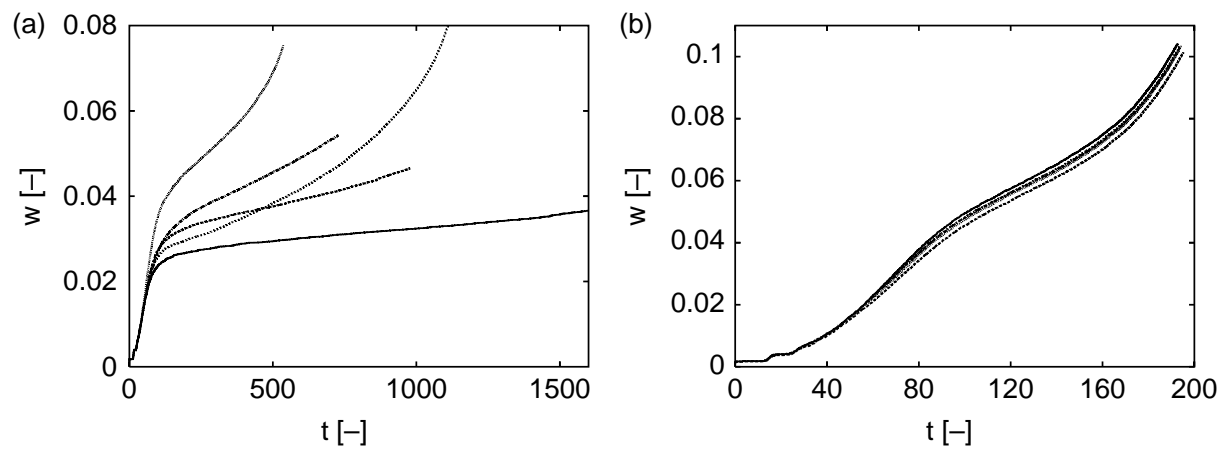

Figure 6. Biofilm occupancy $\omega(t)$ for (a) five simulations in which all inocculation sites were chosen randomly, and (b) for five in which some probiotics were deliberately placed in the inflow region of the flow channel.

plateau phase while the pathogenic population as well as the inerts increase. In cases (b) through (e) the probiotics continue growing while the pathogenic population decreases after an initial growth phase.

While the qualitative behaviour is the same in the cases (b)-(e), the time scales are different. In case (d) the pathogens are depleted after $t \approx 400$, while in case (c) this happens only at $t=1200$. In case (b) the pathogenic population is still considerable at $t=1000$, where it appears to approach a plateau.

The steep increase of the probiotic population in case (c) indicates that a large probiotic biofilm colony develops in the inflow region, where the growth conditions are favourable.

These simulation results indicate that the initial sites of inoculation can affect not only the duration of the eradication process but, indeed, can also determine whether such a control strategy will be successful. To validate this, we conducted another series of five simulations, in which a probiotic colony was deliberately placed in the entrance region of the channel, where growth conditions are most favourable. In Figures 5(f) and 6(b) the lumped parameters are plotted for these simulations. No strong deviations are observed across these simulations. The probiotic colony in the entrance region controls the system behaviour. From this we conclude that in a sparsely inoculated system, like the one under investigation here, the inoculation sites indeed can determine the success of control.

In the probiotic simulation study, the bacteria that are furthest away from the inflow are hit fastest and hardest, while the ones closest to the inflow remain longer under favourable conditions. This is in contrast to what one observes in studies of antibiotic control, where usually the outer rim of the biofilm colonies facing upstream is first neutralized, as e.g. observed in Eberl and Sudarsan [14]. This confirms the findings of the simpler onedimensional study [22], where the growth limiting substrates $C$ and $P$ were controlled from the liquid phase above the biofilm. Mathematically this can be explained by the maximum principle.

\section{Conclusion}

We presented a quasilinear parabolic system of diffusion-reaction system that shows two non-linear diffusion effects: a finite speed of interface propagation due to 'porous medium' degeneracy and an a priori bound on the solution due to a fast diffusion singularity. This system models a mixed-culture biofilm that is formed by a pathogenic 
species and a probiotics species. The probiotics control the pathogens by modifying the living conditions for the pathogens in the environment, in particular by increasing the local concentration of protonated lactic acids and decreasing the local $\mathrm{pH}$. In our modelling study, we considered this biological system in a slow flowing narrow flow channel that is operated under growth favourable inflow conditions for both species.

This model represents a major extension of a previously studied model [22] of suspended probiotics controlling a pathogenic biofilm. Instead of a single doubledegenerate diffusion equation a system of three such equations is obtained, for which we could show that important qualitative properties of a non-standard finite difference method carry over from the better understood case of a scalar equation.

The published literature in probiotic research to date seems rather observational. Therefore, due to a lack of quantitative information (e.g. parameter values, reaction kinetics) at this point most modelling studies of probiotic mechanisms must be to some extent phenomenological, focusing on qualitative model features without necessarily being able to make quantitative predictions. Nevertheless, the numerical simulation studies suggest, at least for sparsely inoculated substrata, that the site of inoculation of the probiotic biofilm colonies might be an important factor determining the long term behaviour of control strategies that are based on modification of $\mathrm{pH}$ and lactic acid concentrations.

In addition to the more complex multi-species interaction and the explicit account of the hydrodynamic contribution to mass transfer, a major difference between this study and the previous simulation study of biofilm control by a suspended probiotic culture, is the following: In our 2D simulations we considered a sparse colonization of the susbtratum with biofilms of either kind. The 1D description in Khassehkhan and Eberl [22], however, implied that a dense and uniform pathogenic biofilm covers the substratum. One of the major findings of the earlier study was that the cells close to the substratum are deactivated first, in contrast to antibiotic disinfection where usually the cells at the biofilm/water interface are inactivated first, while the bacteria in the inner layers might be able to survive virtually unharmed. As expected from these previous results, the maximum principle, and the consideration of convective transport of harmful substances downstream, we found in our 2D simulations that the (isolated) biofilm colonies in the downstream regions are deactivated first while often the upstream colonies may remain under favourable growth conditions. Again, this is in contrast to the results of the antibiotic disinfection results of Eberl and Sudarsan [14], in which the opposite was found. This might suggest to test for a combination of probiotic (modification of $\mathrm{pH}$, lactic acids) and antibiotic treatment. Including this into one mathematical model will further increase computational complexity but should be possible and relatively straightforward under the modelling framework used here.

\section{Acknowledgements}

This study was supported in parts by Canada's Network Centers of Excellence programme through the Advanced Foods and Material Network (AFMNET). HJE acknowledges also the support received from NSERC (Discovery Grant) and the Canada Research Chairs Program.

\section{References}

[1] R. Anguelov and J.M.S. Lubuma, Contributions to the mathematics of the nonstandard finite difference method and its applications, Numer. Methods Partial Differential Equations 17 (2001), pp. 518-543. 
[2] K. Anguige, J.R. King, J.P. Ward, and P. Williams, Mathematical modelling of therapies targeted at bacterial quorum sensing, Math. Biosci. 192(1) (2004), pp. 39-83.

[3] K. Anguige, J.R. King, and J.P. Ward, Modelling antibiotic- and quorum sensing treatment of a spatially-structured Pseudomonas aeruginosa population, J. Math. Biol. 51 (2005), pp. 557-594.

[4] G.K. Batchelor, An Introduction to Fluid Dynamics, Cambridge University Press, Cambridge, UK, 1968.

[5] A. Bezkorovainy, Probiotics: determinants of survival and growth in the gut, Am. J. Clin. Nutr. 73S (2001), pp. 399-405.

[6] F. Breidt and H.P. Flemming, Modeling of the competitive growth of Listeria monocytogenes and Lactococcus lactis in vegetable broth, Appl. Environ. Microbiol. 64(9) (1998), pp. 3159-3165.

[7] CIHR Institute of Infection and Immunity (III), Novel alternatives to antibiotics, Workshop Report, 2005, available at http://www.cihr-irsc.gc.ca/e/27879.html.

[8] J.W. Costerton, P.S. Stewart, and E.P. Greenberg, Bacterial biofilms: a common cause of persistent infections, Science 248(5418) (1999), pp. 1318-1322.

[9] L. Demaret, H.J. Eberl, M.A. Efendiev, and R. Lasser, Analysis and simulation of a meso-scale model of diffusive resistance of bacterial biofilms to penetration of antibiotics, Adv. Math. Sci. Appl. 18 (2008), pp. 269-304.

[10] B.C. Dunsmore, A. Jacobsen, L.H. Stoodley, C.J. Bass, H.M. Lappin-Scott, and P. Stoodley, The influence of fluid shear on the structure and material properties of sulphate-reducing bacterial biofilms, J. Ind. Microbiol. Biotech. 29 (2002), pp. 347-353.

[11] A. Duvnjak and H.J. Eberl, Time-discretisation of a degenerate reaction-diffusion equation arising in biofilm modeling, Electron. Trans. Num. Anal. 23 (2006), pp. 15-38.

[12] H.J. Eberl and L. Demaret, A finite difference scheme for a doubly degenerate diffusionreaction equation arising in microbial ecology, Electron. J. Differential Equations CS 15 (2007), pp. 77-95.

[13] H.J. Eberl and H. Schraft, A diffusion-reaction model of a mixed culture biofilm arising in food safety studies, in Mathematical Modeling of Biological System, A. Deutsch, et al., eds.,vol. II, Birkhäuser, Boston, MA, 2007, pp. 109-120.

[14] H.J. Eberl and R. Sudarsan, Exposure of biofilms to slow flow fields: the convective contribution to growth and disinfections, J. Theor. Biol. 253(4) (2008), pp. 788-807.

[15] H.J. Eberl, D.F. Parker, and M.C.M. van Loosdrecht, A new deterministic spatio-temporal continuum model for biofilm development, J. Theor. Med. 3(3) (2001), pp. 161-175.

[16] M.A. Efendiev and L. Demaret, On the structure of attractors for a class of degenerate reaction-diffusion systems, Adv. Math. Sci. Appl. 18 (2008), pp. 105-118.

[17] M.A. Efendiev, H.J. Eberl, and S.V. Zelik, Existence and longtime behavior of solutions of a nonlinear reaction-diffusion system arising in the modeling of biofilms, RIMS Kokyuroko 1258 (2002), pp. 49-71.

[18] M.A. Efendiev, S.V. Zelik, and H.J. Eberl, Existence and longtime behavior of a biofilm model, Comm. Pure Appl. Anal. 8(2) (2009), pp. 509-531.

[19] FAO/WHO Working Group, Guidelines for the Evaluation of Probiotics in Food, FAO/WHO, London, ON, 2002.

[20] W. Hackbusch, Theorie und Numerik elliptischer Differentialgleichungen, Teubner, Stuttgart, 1986.

[21] H. Khassehkhan and H.J. Eberl, Interface tracking for a non-linear, degenerated diffusionreaction equation describing formation of bacterial biofilms, Dyn. Cont. Disc. Imp. Sys. 13A S (2006), pp. 131-144.

[22] H. Khassehkhan and H.J. Eberl, Modeling and simulation of a bacterial biofilm that is controlled by $\mathrm{pH}$ and protonated lactic acids, Comp. Math. Mod. Med. 9(1) (2008), pp. 47-67.

[23] H. Khassehkhan, M.A. Efendiev, and H.J. Eberl, A degenerate diffusion-reaction model of an amensalistic probiotic biofilm control system: existence and simulation of solutions, Disc. Cont. Dyn. Sys. B, in press.

[24] I. Klapper, C.J. Rupp, R. Cargo, B. Purvedorj, and P. Stoodley, A viscoelastic fluid description of bacterial biofilm material properties, Biotech. Bioeng. 80 (2002), pp. 289-296.

[25] K.W. Morton, Numerical Solution of Convection-Diffusion Problems, Chapman \& Hall, London, UK, 1996. 
[26] R.E. Mickens, Nonstandard finite difference schemes, in Applications of Nonstandard Finite Difference Schemes, R.E. Mickens, ed., World Scientific, Singapore, 2000.

[27] J.C. Ochoa, C. Coufort, R. Escudie, A. Line, and E. Paul, Influence of non-uniform distribution of shear stress on aerobic biofilms, Chem. Eng. Sci. 62 (2007), pp. 3672-3684.

[28] S.L. Percival, J.T. Walker, and P.R. Hunter, Microbiological Aspects of Biofilms and Drinking Water, CRC Press, Boca Raton, FL, 2000.

[29] G. Reid, J. Jass, M.T. Sebulsky, and J.K. McCormick, Potential uses of probiotics in clinical practice, Clin. Microbiol. Rev. 16(4) (2003), pp. 658-672.

[30] B.E. Rittmann, The effect of shear-stress on biofilm loss rate, Biotech. Bioeng. 24(2) (1982), pp. 501-506.

[31] Y. Saad, SPARSKIT: a basic tool kit for sparse matrix computations, 1994, http://www-users. cs.umn.edu/saad/software/SPARSKIT/sparskit.html.

[32] M. Saarela, L. Lähteenmäki, R. Crittenden, S. Salminen, and T. Mattila-Sandholm, Gut bacteria and health foods - the European perspective, Int. J. Food Microbiol. 78 (2002), pp. 99-117.

[33] M.E. Sanders, Considerations for use of probiotic bacteria to modulate human health, J. Nutr. 130S (2000), pp. 384-390.

[34] S. Santosa, E. Farnworth, and P.J.H. Jones, Probiotics and their potential health claims, Nutr. Rev. 64(6) (2006), pp. 265-274.

[35] H. Schlichting, Boundary Layer Theory, McGraw Hill, New York, NY, 1968.

[36] P.S. Stewart, Multicellular nature of biofilm protection from antimicrobial agents, in Biofilm Communities: Order from Chaos?, A. McBain, et al., eds., BioLine, Cardiff, 2003.

[37] P.S. Stewart, Diffusion in biofilms, J. Bacteriol. 185 (2003), pp. 1485-1491.

[38] P. Stoodley, R. Cargo, C.J. Rupp, S. Wilson, and I. Klapper, Biofilm material properties as related to shear-induced deformation and detachment phenomena, J. Ind. Microbiol. Biotech. 29(6) (2002), pp. 361-367.

[39] R. Sudarsan, K. Milferstedt, E. Morgenroth, and H.J. Eberl, Quantification of detachment forces on rigid biofiln colonies in a roto torque reactor using computational fluid dynamics tools, Wat. Sci. Tech. 52(7) (2005), pp. 149-154.

[40] B.W. Towler, C.J. Rupp, A.B. Cunningham, and P. Stoodley, Viscoelastic properties of a mixed culture biofilm from rheometer creep analysis, Biofouling 19(5) (2003), pp. 279-285.

[41] J.C. Valdez, M.C. Peral, M. Rachid, M. Santana, and G. Perdigon, Interference of Lactobacillus plantarum with Pseudomonas aeruginosa in vitro and in infected burns: the potential use of probiotics in wound treatment, Clin. Microbiol. Infect. 11(6) (2005), pp. 472-479.

[42] J. Xu, R. Sudarsan, G.A. Darlington, and H.J. Eberl, A computational study of external shear forces in biofilm clusters, 22nd Int. Symp. High Perf. Comp. Sys. Appls. (HPCS 2008) IEEE Proceedings (2008), pp. 139-145. 


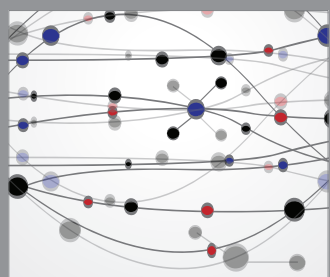

The Scientific World Journal
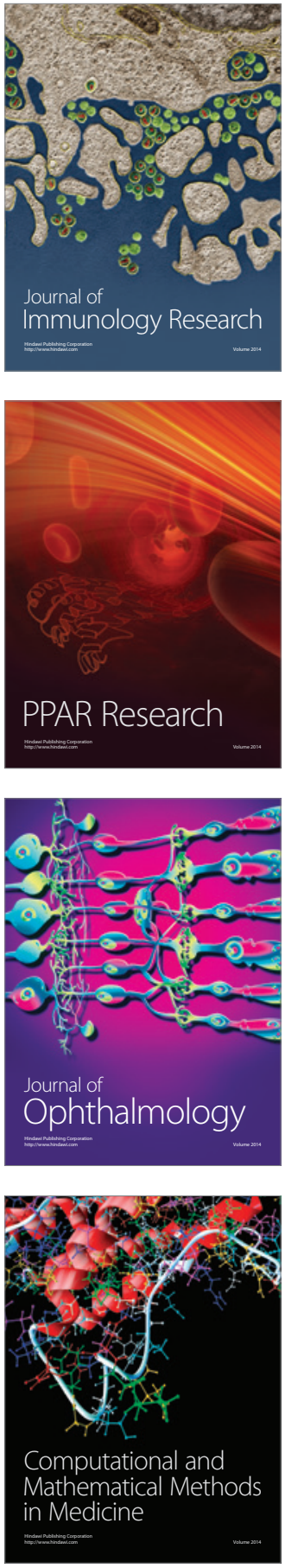

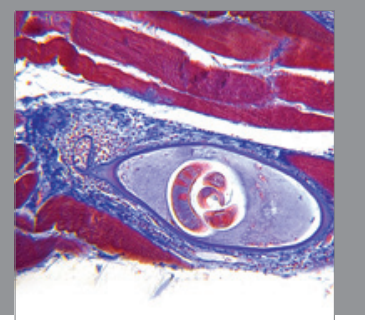

Gastroenterology

Research and Practice
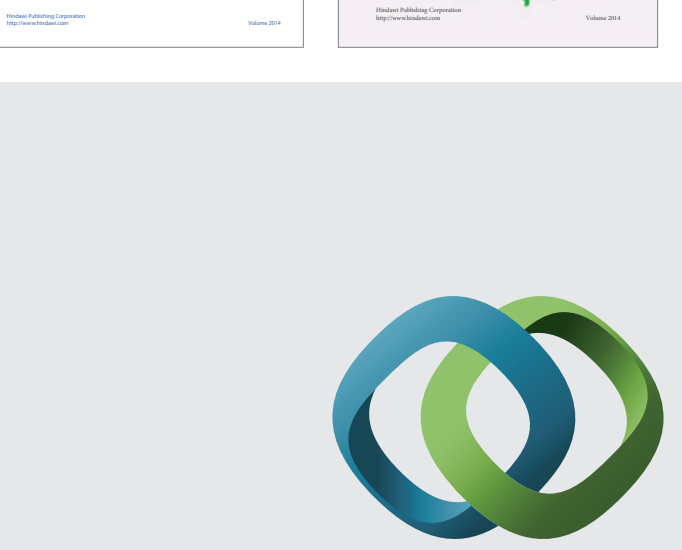

\section{Hindawi}

Submit your manuscripts at

http://www.hindawi.com
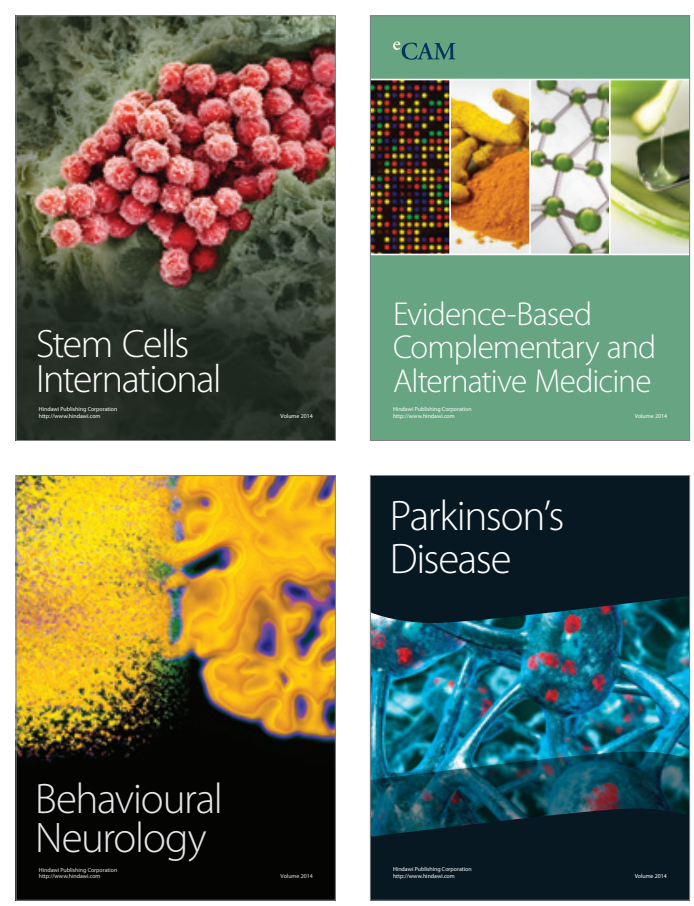

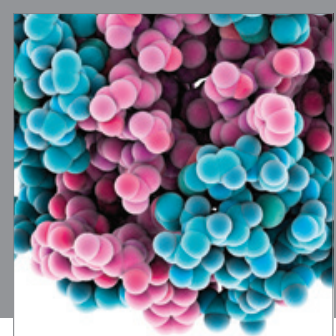

Journal of
Diabetes Research

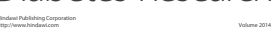

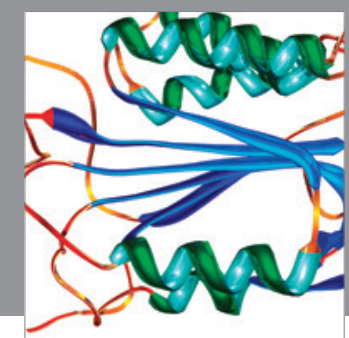

Disease Markers
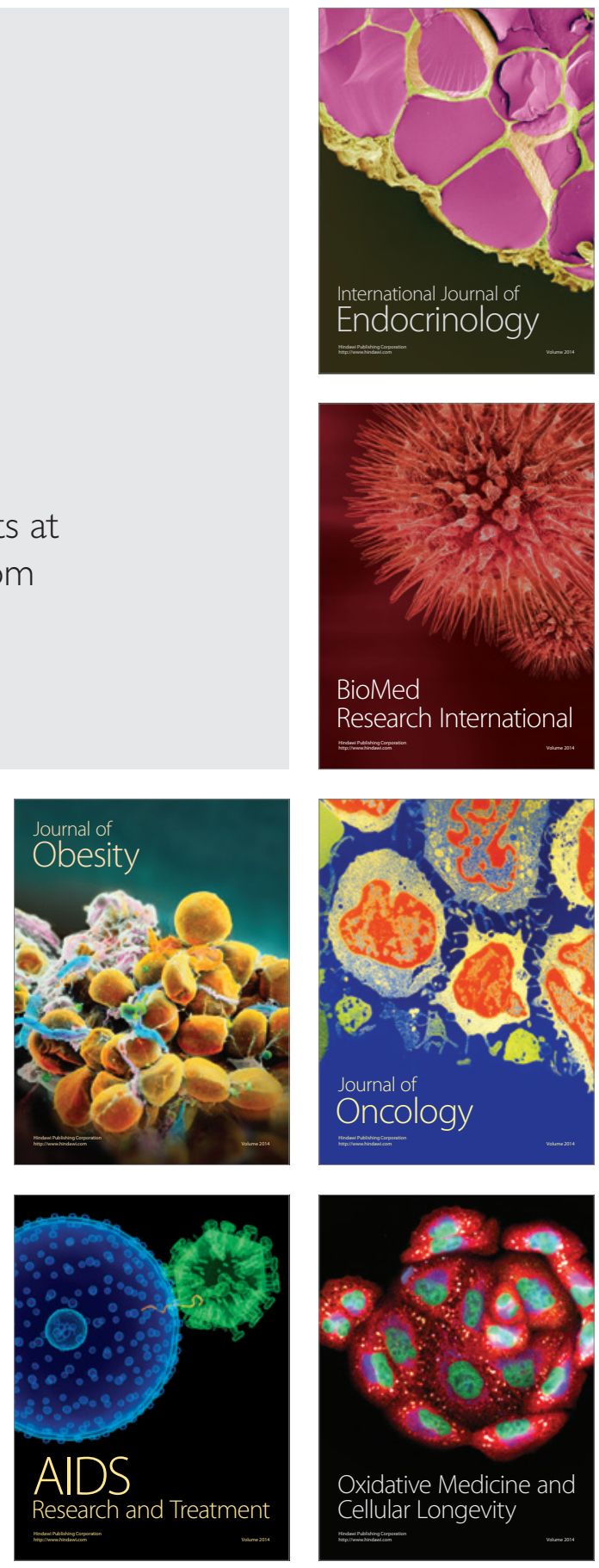